\title{
Wind models and synthetic UV spectra for O-type stars
}

\author{
Tadziu L. Hoffmann, Adalbert W.A. Pauldrach, and Joachim Puls \\ Universitätssternwarte, Ludwich-Maximilians-Universität, \\ Scheinerstraße 1, D-81679 München, BRD
}

\begin{abstract}
Spectral analysis of hot stars requires adequate model atmospheres, which take into account the effects of non-LTE and radiation-driven winds properly. Here we present significant improvements of our approach in constructing detailed atmospheric models and synthetic spectra for O-type stars (model code WM-BASIC; see Pauldrach, Hoffmann \& Lennon 2001). The most important ingredients of these models with regard to a realistic description of stationary winds are: $(i)$ a rigorous treatment of line blocking and blanketing; $(i i)$ a consistent determination of the radiative line acceleration; (iii) a considerably improved and enhanced atomic data archive providing the basis for a detailed multilevel non-LTE treatment and an adequate representation of the radiative line driving; and (iv) the inclusion of EUV and X-ray radiation produced by cooling zones originating from shock-heated matter. This new tool not only allows to constrain the properties of stellar winds, the stellar parameters, and the abundances via a comparison of observed and synthetic spectra, but also provides the astrophysically important information about the ionizing fluxes of these stars. We use this new method to compute selfconsistent wind parameters for a series of Galactic stars analyzed in the optical and verify the theoretically predicted dependence of the wind momentum on metallicity.
\end{abstract}

\section{Comparison with observed values}

The large sample of Galactic O-type stars from Puls et al. (1996) is currently being reanalyzed with an improved model code also including line blanketing effects ( $c f$. Puls et al., these Proceedings); we have computed the selfconsistent wind dynamics for the newly determined stellar parameters. The resulting wind momenta are shown below in the Figure on the left (LC denotes luminosity class). In contrast to the optical analysis by means of unclumped models by Puls et al., the hydrodynamic models do not show evidence of different windmomentum-luminosity relations for different luminosity classes.

We note, however, that for several of the stars the synthetic UV spectrum resulting from the models with the given stellar parameters is incompatible with the observed UV spectrum. While no attempt has been made at this point to reproduce the UV spectra (this would require changes of radii and/or gravities), such a procedure using our consistent hydrodynamic models would, in principle, allow the determination of stellar radii and gravities from the UV spectrum alone (cf. Kudritzki et al. 1992; Hoffmann \& Pauldrach 2002), since both the mass loss rate and the terminal velocity depend on the stellar parameters (surface gravity, radius, and luminosity) through the interplay of radiative forces and gravitation. 

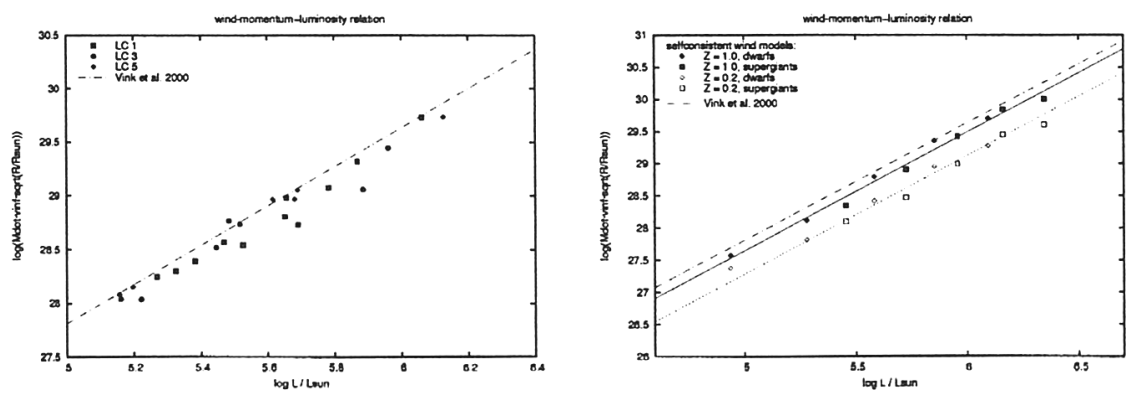

On the other hand, the method by Puls et al. cannot intrinsically determine the radii; they are based on the distances given in the literature and the optical fluxes from the models. Thus, some uncertainty remains in the derived stellar parameters, which we hope to resolve with an analysis of a sample of O-type stars in M31, where the differential error in the distances is negligible.

\section{Dependence of wind momentum on metallicity}

To study the dependence of the wind strengths on metallicity, we have calculated selfconsistent wind models for a grid of stellar models (consisting of a dwarf and a supergiant sequence) at solar and one-fifth solar metallicity. The stellar parameters were chosen so as to reproduce the observed parameters for these types of stars, while at the same time covering a large range in luminosities. Theory predicts that the wind momentum should scale with the metallicity $Z$ as $z^{(1-\alpha) / \alpha^{\prime}}$, where $\alpha-2$ is the exponent of the line strength distribution function and $\alpha^{\prime}$ is the reciprocal of the slope of the wind momentum-luminosity relation. In the Figure on the right, it can be seen that our models quantitatively reproduce this predicted behavior. The filled and open symbols show the calculated wind momenta for solar metallicity and one-fifth solar metallicity stars, respectively. The solid line is a fit to the calculated wind momenta for solar metallicity; the dashed line is this relation scaled by the factor above using a value of $Z=0.2$, yielding wind momenta in good agreement with the calculated ones for $Z=0.2 \mathrm{Z}_{\odot}$. For comparison, we have also plotted the relation (for solar metallicity stars) as found by Vink et al. 2000 (dash-dotted).

Acknowledgments. This work has been supported by the DLR under grant RD-RX-50 OR 99092.

\section{References}

Hoffmann, T.L., Pauldrach, A.W.A. 2002, in: M. Dopita, S. Kwok \& R.S. Sutherland (eds.) Planetary Nebulae: Their Evolution and Role in the Universe, Proc. IAU Symp. No. 209 (San Francisco: ASP), in press

Kudritzki, R.-P., Hummer, D.G., Pauldrach, A.W.A., et al. 1992, A\&A 257, 655

Pauldrach, A.W.A., Hoffmann, T.L., Lennon, M. 2001, A\&A 375, 161

Puls, J., Kudritzki, R.-P., Herrero, A., et al. 1996 A\&A 307, 171

Vink, J.S., de Koter, A., Lamers, H. 2000 A\&A 362, 295 